\title{
Establishment of a Clinicopathological Database for Traumatic Human Spinal Injury
}

\author{
A. Woods, Dip Tech (WA), E. R. Smith, BSc (Hons), U. Gaekwad, MBBS, MS \\ (GS), MS (Neuro), B. A. Kakulas, AO, MD, (Hon. Athens), MD (WA), \\ FRACP, FRC Path, FRCPA \\ Department of Neuropathology, Royal Perth Hospital and Neuromuscular Research \\ Institute, University of Western Australia, Perth, Australia.
}

\section{Summary}

The establishment of a comprehensive retrospective clinicopathological database of patients who died with traumatic spinal injury is described. All necropsy patients examined from 1957 onwards, at the Royal Perth Hospital (RPH) with either a spinal cord or spinal column injury or both were included in the study. There are two broad categories of material. Firstly, those patients with a spinal cord injury (SCI) who survived the initial episode and were treated in the Sir George Bedbrook Department of Paraplegia, RPH. Secondly there are those who suffered either a vertebral column or SCI and died immediately, referred by the Coroner. In most cases, in both groups the spinal injury was not the direct cause of death. The catchment area includes the entire state of Western Australia. The database is essentially a neuropathological resource and is not intended to have any direct epidemiological implications. The information in the database is available for clinicopathological research such as:

(a) To assist the clinician in planning the bedside management of patients with SCI.

(b) To assess and quantify preserved anatomical structures for correlation with residual physiology in human SCI as a foundation for restorative neurology.

(c) To define the spinal cord lesion in detail as a framework for the application of the many recent advances in basic neurobiology toward the reconstruction of the injured spinal cord.

(d) To provide clinical data for use in conjunction with the tissue bank of spinal injuries established at $R P H$, in further research.

Key words: Traumatic spinal injury; Human spinal injury; Database; Computer; Data collection.

We report the establishment of an extensive computerised database containing personal, clinical and pathological information. The database includes entries detailing acute fatal spinal injuries where the patient either died immediately as a result of the spinal injury or as a result of associated injuries received concurrently, together with clinicopathological details of chronic injuries in survivors. The 
inclusion of acute as well as chronic cases in this study gives a better comparison of the human spinal cord lesion with observations derived from experimental work in animals.

Several data collection systems have been established by others to analyse certain aspects of spinal injuries. Burke et al. (1985), established their system to assess cause of accident, epidemiological information and clinical outcome of treatment. Burke et al. (1987), assessed urological outcome while Kewalramani et al. (1980), were involved in an epidemiological and clinical study of spinal cord injuries (SCI) in pediatric populations. However this is the only database linked to a tissue bank of human SCI.

The reasons for the establishment of this database were:

(1) To assist the clinician in planning bedside management.

(2) To identify epidemiological factors associated with SCI, within the limitations of the system.

(3) To assess important clinicopathological correlations such as the quantification of preserved spinal cord tissues, for material stored in the RPH tissue bank, with residual clinical and neurophysiological functions.

(4) To assist in the selection of cases for further research.

(5) To enable where possible correlations to be made to identify factors involved in the development of complications and finally the prediction of survival.

\section{The computer system}

An IBM PC-AT was chosen because of its large storage capacity and speed. This multi function machine hosts a database management system, word processing package, graphics package and other utilities which enable diskettes from foreign machines to be read. The program, dBASE III PLUS by Ashton Tate, was chosen for the project to store the relevant data. The program allows a variety of manipulative functions essential for later analysis. Microsoft Chart Version 3 is used to present the information in graphic form.

Files are stored on the hard disc drive within the computer and regular backup copies are stored in a fireproof safe for security against physical damage and for patient confidentiality.

\section{Creation of the database}

Four steps were taken to construct the database:

(1) Design

(2) Creation of data collection sheets

(3) Extraction of data from the case records

(4) Entry and manipulation of data

\section{Designing the structure}

Primary information was collected from the files in the Department of Neuropathology, Royal Perth Hospital (NRPH), the Perth City Coroners Department 
and the Royal Perth Rehabilitation Hospital (RPRH). The pathological data came primarily from NRPH, while the patients clinical notes were principally from RPRH. Following consultation with clinicians working in the area of spinal trauma management the data was divided into eight database files to ensure quick and easy access to the large amount of information available in the initial 312 patient records to be entered.

\section{Data sheets}

Data sheets, on which all patient histories were entered were designed to parallel the data base files for ease of data entry. They also served as a back up hard copy.

As the information was collected each case record was entered into the computer. Data entry continues as new records become available.

\section{Structure of the database files (DBF)}

A description of each of the eight database files is given below. In order to retrieve the information each category must contain information which is organised in a standard form that is either logical, numerical or descriptive. The combined eight databases consist of 298 fields, containing 6484 characters.

\section{First database}

The first database file consists of basic information. The name, surname, sex, age at accident and address of each patient is listed as well as birthdate and birthplace. Occupation is also included to assess incidence related to work practices (the Appendix shows page one of the data collection sheet used to obtain this information). Marital status is listed because there is a high incidence of marital breakdown among paraplegic or quadriplegic patients. The clinical assessment of the patient is entered as paraplegia or quadriplegia. X-ray results indicate the extent of damage to the spinal vertebrae and the presence of any displacement or deformity. All injuries to the spinal column are recorded, including multiple fractures. Details of the injury to the cord, including the cauda equina, are recorded, the level of injury and whether the lesion was a partial or a complete transection. If the lesion is partial comments are made on the distribution of the lesion e.g. central cord necrosis, anterior cord syndrome, posterior column sparing, posterior column lesion.

Death date and cause of death, taken from the post-mortem examination is categorised and a special note is made if the cause of death was directly related to the spinal injury.

Patient's doctor, unit number and neuropathology file number are also included.

\section{Second database}

The acute period

All details of the accident are included, the date and time, and the presence of drugs and/or alcohol. The cause of the accident is broadly categorised into groups: 
Road Accident

Work Accident

Aquatic Accident

Train Accident

Fall

Flying Accident

Gunshot

The second database (Two.dbf) also includes details of any associated injuries of the skull, brain, chest, abdomen and long bones observed at the scene of the accident. Any treatment given is noted. The sensory and motor levels of the patient and any movement of limbs is included, including a silent cauda equina lesion resulting in permanent lower motor neuron bladder dysfunction. Also the (possible) presence of a second vertebral fracture. Any lapses of consciousness are recorded, along with the duration of those lapses.

\section{Third database}

\section{The local Hospital}

The date and time of admission to the local hospital is noted, as is the patients motor and sensory levels, level of consciousness and blood pressure. Reflexes on both sides of the body are listed with X-ray diagnosis and final diagnosis. Haematology and biochemistry results such as Haemoglobin, $\mathrm{PO}_{2}, \mathrm{PCO}_{2}, \mathrm{WBC}$, ESR, blood urea, blood sugar and serum electrolytes are included. Any treatment administered at the local hospital is recorded and importantly the immediate status of the patient is noted, with observations of any improvement or deterioration in condition.

\section{Fourth database}

\section{The major hospital}

The database contains the same categories as Three.dbf but in reference to the major hospital.

\section{Fifth database}

The fifth database contains the same categories as the third and fourth database files, but in relation to the time spent in the spinal unit (RPRH). Five.dbf includes additional information with particular reference to whether the patient suffers from a clinically complete or incomplete SCI. Notes on treatment such as; traction, cervical collar, manual reduction, operative reduction, postoperative reduction, Harrington rods and laminectomy are also included.

\section{Sixth database}

The sixth database file includes information on the condition of the patient after treatment. Reflexes and change in condition are noted. Complications are listed in eight separate groups for comment. 
—urinary tract infection

- pulmonary complications

- vascular complications (including deep vein thrombosis)

-joint complications (including heterotopic ossification with marked limitation of joint function)

- psychiatric complications

-pressure sores

- phantom pains

- burning sensations

-spasm

- distortion of body image

\section{Seventh database}

The seventh database describes the condition of the patient at the time of discharge. Reflexes, X-ray, bladder function are all mentioned as in the previous database files. Any urological or plastic surgery is noted. Marital status is reassessed together with employment, sexual function and change in condition. Each patient is put in a category according to Frankel's method of classification.

\section{Eighth database}

Eight.dbf describes the neuropathology of each patient (the Appendix contains a page from the data collection sheet detailing this data). Information contained here includes; the level of bone and/or disc damage, the type of injury e.g. flexion, extension, any deformity or narrowing of the spinal canal and any damage to the spinal cord. The condition of the cord is fully described including documentation on areas of compression, atrophy, demyelination, softening and level and extent of the lesion. The type of lesion whether pathologically complete or incomplete is included. A brief description of the condition of the dura and arachnoid mater, the two spinal ligaments and the state of the arteries and veins, including details of any haemorrhage is added. A detailed account of the remaining parenchyma and the distribution of this tissue is also included, together with a description of regenerating fibres and their origin.

\section{Discussion}

One of the limitations of this study is its mainly retrospective nature, this naturally limits the amount of information available in each case. The ideal is, of course, the establishment of a prospective study of all cases from the time of accident to their final outcome. It is hoped that such a study will soon be commenced throughout Australia. However, the authors believe that a vast amount of knowledge for people associated with management of spinal trauma patients is still assessable through the thorough examination of past patient histories.

\section{Conclusions}

The information contained in the database has already been shown to be extremely 
useful and a number of projects have already arisen in this way (Kaelan et al., 1988). It has been invaluable in the quick and accurate selection of cases for further clinicopathological research. Other important questions are presently being assessed; anatomical correlations are being analysed to determine factors influencing the neurological status and natural history of the disorder.

\section{Acknowledgements}

This study was supported by a grant from Medical Research Foundation of Western Australia (MEDWA). We would like to thank Sir George Bedbrook for his assistance and encouragement, Mr P. Morling for his assistance with the computer and Mr P. Jacobsen, for his assistance with the manuscript.

\section{References}

BURKe DC, BURLEY T, UNGAR GH 1985 Data on spinal injuries-Part I. Collection and analysis of 352 consecutive admissions. Australian and New Zealand fournal of Surgery 55:3-12.

BURKe DC, BURLEY T, UNGAR GH 1985 Data on spinal injuries-Part II. Outcome of the treatment of 352 consecutive admissions. Australian and New Zealand Fournal of Surgery 55:377-382.

BURKE DC, BROWN DJ, BURLEY HT, UNGAR GH 1987 Data collection on spinal cord injuries: Urological outcome. Paraplegia 25:311-317.

KAELAN C, JACOBSEN PF, KAKULAS BA 1988 An investigation of possible transynaptic neuronal degeneration in human spinal cord injury. Fournal of the Neurological Sciences 86:231-237.

KEWALRAMANI LS, KRAUS JF, STERLING HM 1980 Acute spinal-cord lesions in a pediatric population: epidemiological and clinical features. Paraplegia 18:206-219.

\section{Appendix I \\ Spinal Injury Research Programme \\ Chronic Spinal Injury Basic Information}

Surname:

Christian names:

Date of birth:

Country of birth:

Age: Sex: Occupation:

Marital status:

Address:

Date of accident:

Age at accident:

Clinical diagnosis:

X-ray

Spinal Cord Level

Complete Lesion

Partial Lesion

- Anterior Cord Syndrome

- Central Cord Syndrome

-Posterior Column Sparing

-Posterior Cord Syndrome

-Brown - Sequard 
Date of death:

Cause of death:

Related to spinal injury: Yes No

Survival period:

Stored cord:

Stored column:

Doctor:

Unit No:

Neuropathology number:

\section{Neuropathology}

Spinal column

Level of fracture

Type of fracture-Compression Flexion Flexor-rotation Extension Extension-rotation Transverse

Ligaments: Anterior Longitudinal Ligament-intact/ruptured Posterior Longitudinal Ligament-intact/ruptured

Deformity

Spinal canal Normal Narrowed \%

$\begin{array}{lll}\text { Spinal cord } & \text { Level Extent }\end{array}$

Extradural haemorrhage

Subarachnoid haemorrhage

Normal cord

Compression

Adhesions

Atrophy

Partial loss of cord substance

Total loss of cord substance 